\title{
Elaboración de ladrillos con merma de una empresa que fabrica espumas reticuladas
}

\section{Elaboration of bricks with merma from a company that manufactures reticulated foams}

\author{
MARTÍN-DEL CAMPO, Ma. Guadalupe*†, HERNÁNDEZ, Gabriela y GÓMEZ, Guillermina \\ Universidad Tecnológica del Valle de Toluca, Carrera de Tecnología Ambiental
}

ID $1^{\mathrm{er}}$ Autor: Ma. Guadalupe, Martín-Del Campo / ORC ID: 0000-0003-2689-1684

ID $1^{\text {er }}$ Coautor: Gabriela, Hernández / ORC ID: 0000-0002-7601-5263

ID $2^{\text {do }}$ Coautor: Guillermina, Gómez / ORC ID: 0000-0002-3621-2262, CVU CONACYT ID: 93419

DOI: $10.35429 /$ JCE.2019.9.3.7.11

Recibido 03 de Junio, 2019; Aceptado 30 Septiembre, 2019

\begin{abstract}
Resumen
Se desarrolló una propuesta para elaborar ladrillos a base de merma molida de una empresa que se dedica a la fabricación de espumas reticuladas, el proceso consistió en establecer primeramente las dimensiones acordes a lo que está actualmente en el mercado, se determinó un blanco de referencia con las concentraciones similares a un ladrillo artesanal, enseguida la sustitución de las concentraciones del blanco para la prueba A fueron de $40 \%$ de tepojal, $30 \%$ de cemento, $20 \%$ de arena y $10 \%$ de merma y la prueba $\mathrm{B}$, de $45 \%$ de tepojal, $35 \%$ de arena, $20 \%$ de merma, para la evaluar la factibilidad técnica en la elaboración, de las especificaciones de la NMX-C441-ONNCCE, como resistencia a la compresión, absorción de agua y las dimensiones, así como la densidad de los ladrillos elaborados, el cual se obtuvo como resultado que el de la prueba $\mathrm{B}$ es muy viable para el uso no estructural es decir muros divisorios, ya que soporta una carga de $1000 \mathrm{~kg}$, son eficientes para colocarlos a la intemperie ya que el porcentaje de absorción de agua es del 4\%, así como la factibilidad económica, con un costo de \$13.1.
\end{abstract}

Ladrillo, Merma, Resistencia

\begin{abstract}
A proposal was developed to make bricks based on ground shrinkage of a company that is dedicated to the manufacture of cross-linked foams, the process consisted of first establishing the dimensions according to what is currently in the market, a reference target was determined with concentrations similar to an artisanal brick, immediately replacing the white concentrations for test A were $40 \%$ of tepojal, $30 \%$ of cement, $20 \%$ of sand and $10 \%$ of shrinkage and test B of $45 \%$ of Tepojal, $35 \%$ sand, $20 \%$ depletion, to evaluate the technical feasibility in the elaboration of the specifications of NMX-C-441ONNCCE, such as compressive strength, water absorption and dimensions, as well as The density of the elaborated bricks, which was obtained as a result of that of test B is very viable for non-structural use, that is to say dividing walls, since it supports a load of $1000 \mathrm{~kg}$, they are efficient to place them at weathering since the percentage of water absorption is $4 \%$, as well as the economic feasibility, with a cost of $\$ 13.1$.
\end{abstract}

Brick, Shrinkage, Resistance

Citación: MARTÍN-DEL CAMPO, Ma. Guadalupe, HERNÁNDEZ, Gabriela y GÓMEZ, Guillermina. Elaboración de ladrillos con merma de una empresa que fabrica espumas reticuladas. Revista de Ingeniería Civil. 2019. 3-9: 7-11

\footnotetext{
* Correspondencia del Autor (correo electrónico: maria.martindelcampo@utvtol.edu.mx)

$\dagger$ Investigador contribuyendo como primer autor.
} 


\section{Introducción}

El problema de los desechos industriales que genera la actividad económica diaria en México es uno de los temas principales que se enfrenta el ambiente, y a lo largo del tiempo disminuir las toneladas que se desechan diariamente genera costos elevados y de alguna forma contaminación ya sea en el agua, suelo o aire.

La empresa donde se desarrolló el proyecto es considerada de alto riesgo de incendio, debido a las toneladas de residuos que se generaron a lo largo de la producción de las espumas como su actividad principal, así como las múltiples multas y sanciones que se pueden generar por parte de organismos gubernamentales por la mala disposición, a consecuencia de esto es necesario encontrar una alternativa para la disminución de los residuos y que ayude a mitigar estos riesgos.

Atendiendo estas causas se realizó una identificación de los riesgos a los que se encuentra la empresa por la cantidad de residuos acumulados, se propuso la elaboración de ladrillos sustentables hechos de la merma y se colocaron en forma de barrera en ciertos puntos de la empresa, para evaluar su durabilidad a las condiciones del ambiente y lograr la disminución de la merma, así como el cumplimiento y regulación a la legislación y normatividad ambiental. Los beneficios que obtendrá la empresa se reflejarán en el análisis de los costos con base a la disminución de la merma

\section{Residuos de EVA}

El Etil-vinil-acetato (EVA) son copolímeros que obtienen por reacción a alta presión entre los monómeros etileno y acetato de vinilo, durante la fabricación de los productos, son generados inevitables residuos debido a recortes de planchas de EVA para obtener los formatos deseados. Del total de residuos generados, sólo un $20 \%$ se reutiliza en el proceso de producción de nuevos productos debido a las limitaciones de sus características físicas. Por lo tanto, la solución a un destino adecuado del restante de los residuos ha sido objeto de preocupación, llevando a la búsqueda de una alternativa para el destino más común, que es la utilización del potencial de energía de los restos de EVA para alimentar los hornos de las industrias de cemento. (Hax; et al, 2000)
El residuo de EVA es un material de baja densidad, gran capacidad de deformación, tiene buenas características térmicas y acústicas, es estable, inerte y no susceptible a los hongos. (Melo y Silva, 2013)

\section{Propuestas de disposición de EVA}

Las propuestas que se tienen para la disminución de merma de EVA se encuentran: Utilizar la merma como "geo membrana o geotextil", es decir colocar los residuos en la superficie para evitar la percolación de los líquidos en un relleno sanitario, realizando una molienda de la merma y hacer láminas mediante la vulcanización, se utiliza para evitar que haya fisuras en la membrana por los residuos sólidos. (Bouazza y Zornberg, 2019)

Aprovechar los residuos para disponerlos en un relleno sanitario, realizando la molienda de la merma y utilizarlo en conjunto con la tierra, para la compactación de los residuos. (Zattera, et, al., 2005). Elaborar ladrillos a base de la merma, con el fin de utilizarlos como uso no estructural, por medio de la molienda y aplicación de ingeniería. (Melo y Mendoca, 2017)

Es sabido que residuos EVA tienen un gran potencial de utilización como agregados ligeros, reemplazando a los áridos naturales en la fabricación de productos alternativos. Investigaciones realizadas en Brasil han demostrado la viabilidad técnica para el reciclaje de estos residuos. Este trabajo presenta una evaluación de las especificaciones que debe cumplir conforme a la NMX-C-441-ONNCCE2005, Industria de la construcción - bloques, ladrillos o tabiques y tabicones de concreto, para uso no estructural, que se refiere a los mismos que se utilizan en la construcción para muros de relleno, de revestimiento, interiores o exteriores o para cualquier uso no estructural.

La propuesta de éste trabajo ha sido desarrollada en países como Estados Unidos y Brasil, de acuerdo a la información recopilada, dicho lo anterior se basa en la realización de ladrillos a base de merma molida que contiene compuestos de EVA y polietileno y materiales como cemento, arena y tepojal principalmente, con el fin de utilizarlos para las construcciones. 
Al elaborar el ladrillo se consideró aplicable para utilizarlo en la empresa, al principio consistirá en realizar pruebas, elaborando estructuras y colocarlas en diferentes puntos de la empresa, para comprobar su durabilidad a la intemperie, por las propiedades de la merma, de esta manera se evaluará el costo que se tiene por los materiales a adquirir y el costo de molienda, además se pretende conocer el beneficio a largo plazo.

\section{Metodología a desarrollar}

\section{Elaboración de ladrillos}

La elaboración de los ladrillos fue de la siguiente manera:

Molienda y tamizado de la merma: La selección del material para moler fueron tiras sobrantes del corte de los bloques, las cuales fueron colocadas en el molino, posteriormente se hicieron pasar a un silo donde se tamizó una parte y la otra pasó a un silo de almacenamiento; la cantidad que se obtiene es de 30 a $35 \mathrm{~kg}$ por hora, por lo que en un periodo de 8 horas se obtiene hasta $280 \mathrm{Kg}$ de merma en polvo. Para realizar las diferentes pruebas se determinó el tamaño de partícula del molido por medio del tamizado. El material molido se pasó por tres tamices del número 6, 10 y 35. Éste último fue un polvo de $0.5 \mathrm{~mm}$ de diámetro con el que se obtiene una mejor compresión de la mezcla de los materiales.

Materiales para el ladrillo: Se definieron los materiales empleados como materia prima, lo que son, el tepojal, la arena y el cemento, se realizó la obtención y el traslado de estos materiales al lugar acondicionado para trabajar y materiales como el molde, recipientes, palas, etc.

Moldeo: Se diseñaron las concentraciones correspondientes para la elaboración un blanco de referencia y las pruebas y dos pruebas con diferentes porcentajes de material de mezcla y la merma.

Secado: Se determinó el tiempo de secado al sol y posteriormente el secado a horno para eliminar la humedad restante.

Posteriormente a la elaboración de ladrillos, se prosiguió con las evaluaciones de acuerdo a la normatividad correspondiente a la industria de la construcción.

\section{Evaluación}

En relación con los ladrillos elaborados se llevó a cabo la evaluación de las especificaciones a cumplir, conforme a la norma de la construcción NMX-C-441-ONNCCE-2005, Bloques, ladrillos o tabiques y tabicones de concreto, para uso no estructural y de acuerdo a las normas para el método de prueba, el cual se puede visualizar en el Anexo 3

1. Determinación de dimensiones. (NMXC-038-ONNCCE, 2013)

2. Absorción del agua. (NMX-C-037ONNCCE, 2013)

3. Resistencia a la comprensión. (NMX-C036-ONNCCE, 2013)

Mediante los datos obtenidos en la evaluación, se determinó cual es el ladrillo que cumple con las especificaciones, de acuerdo a las tablas que enuncia la norma (NMX-C-441ONNCCE, 2005).

Dicho lo anterior se realizó el análisis de costos involucrados, para determinar si es viable la introducción del prototipo al mercado

\section{Análisis de costos}

Se llevó a cabo el análisis de costos considerando lo siguiente:

Materia Prima: Se determinó los costos de la materia prima involucrada en la elaboración el ladrillo, es decir el tepojal, el cemento, arena y agua, conforme a las cantidades que se utilizaron, de cada una de las pruebas

Proceso de Molienda de merma: Se analizaron los costos que se generan por cierta cantidad de merma molida, de acuerdo a los componentes del molino, durante un periodo de tiempo y el costo que se invierte por la mano de obra involucrada del personal. Análisis y Comparación de Costos: Se realizó una comparación de acuerdo a la demanda de ladrillos en el mercado y los costos obtenido de los ladrillos de merma

\section{Resultados}

Ladrillo generado. Se obtuvieron tres tratamientos (ladrillos con diferentes porcentajes de materiales (Tabla 1).

MARTÍN-DEL CAMPO, Ma. Guadalupe, HERNÁNDEZ, Gabriela y GÓMEZ, Guillermina. Elaboración de ladrillos con merma de una empresa que fabrica espumas reticuladas. Revista de Ingeniería Civil. 2019 


\begin{tabular}{|c|c|c|c|}
\hline Materiales & Blanco & Ladrillo A (\%) & $\begin{array}{c}\text { Ladrillo } \\
\text { B (\%) }\end{array}$ \\
\hline Tepojal & 45 & 40 & 45 \\
\hline Cemento & 35 & 30 & 35 \\
\hline Arena & 20 & 10 & \\
\hline Merma & & 20 & 20 \\
\hline
\end{tabular}

Tabla 1 Porcentaje (\%) de la mezcla de los materiales

\section{Evaluación de los ladrillos}

Los primeros puntos a evaluar fueron las dimensiones el cuál la Norma NMX-C-038ONNCCE, establece el método de prueba para la determinación de las dimensiones del ladrillo; se utilizó una regla para realizar la medición. Con las dimensiones de fabricación planteadas se corroboró que las dimensiones de fabricación cumplen con los lineamientos establecidos en la norma de calidad NMX-C-441-ONNCCE-2013. Posteriormente se calculó la densidad de los materiales, con el objetivo de conocer la cantidad de masa sobre el volumen de cada una de las pruebas, haciendo una comparación con las densidades reales obtenidas de un manual de construcción, el cual las densidades están muy por debajo de los $1500 \mathrm{~kg} / \mathrm{m} 3$ los resultados se muestran en la Tabla 2.

\begin{tabular}{|c|c|c|}
\hline Prueba & Masa (g) & $\begin{array}{l}\text { Densidad } \\
\left(\mathrm{kg} / \mathrm{m}^{3}\right)\end{array}$ \\
\hline $\begin{array}{l}\text { *Ladrillos, tabiques } \\
\text { y tabicones }\end{array}$ & - & $* 1500-1700$ \\
\hline Blanco & 5570 & 1519.44 \\
\hline Ladrillo A & 3999 & 985.18 \\
\hline Ladrillo B & 4300 & 1061.72 \\
\hline
\end{tabular}

*Clasificación de piezas de acuerdo a los materiales empleados en su fabricación NMX-C-441-ON*Densidad obtenida de acuerdo al Manual de constructor

Tabla 2 Densidad de los ladrillos elaborados

Con respecto a la determinación inicial de la NMX-037-ONNCCE-2013, el ladrillo B cumplió las especificaciones. Los valores obtenidos de la resistencia a la compresión no cumplieron con el valor normativo de $25 \mathrm{~kg} / \mathrm{cm}^{2}$ establecido por la NMX-C-036-ONNCCE2012, de igual manera no cumplieron con el límite superior de absorción inicial de agua de la NMX-C-441-ONNCCE-2013 las pruebas estuvieron por arriba de los $5 \mathrm{~g} / \mathrm{min}$ que marca la norma. El uso de ladrillos de la mezcla B en la construcción, como muros no estructurales que no soportan cargas mayores a una tonelada (muros divisorios, bardas o incluso con fines estéticos) pueden ser recomendables.

\section{Análisis de costo}

En la Tabla 3 se muestra el comparativo del costo de los ladrillos donde el Block macizo ligero tiene un precio de $\$ 15.38$, y es utilizado solo para muros divisores, jardineras, etc.; contrariamente el Block macizo gris cuyo precio es de \$11:00 su uso es estructural, es decir para edificaciones, viviendas y muros de carga

\begin{tabular}{|l|l|l|}
\hline Pieza & \multicolumn{1}{|c|}{$\begin{array}{l}\text { Costo ( } \\
\text { \$) }\end{array}$} \\
\hline $\begin{array}{l}\text { Block Macizo } \\
\text { Gris }\end{array}$ & $11.00^{*}$ & \\
\hline $\begin{array}{l}\text { Block Macizo } \\
\text { Ligero }\end{array}$ & $15.38^{*}$ & \\
\hline Ladrillo Blanco & 10.81 & \\
\hline Ladrillo A & 12.46 & \\
\hline Ladrillo B & 13.10 & \\
\hline
\end{tabular}

Tabla 3 Comparación de precios de los ladrillos comerciales y elaborados

*Fuente: Construrama México

Con respecto a el análisis por unidad de ladrillo elaborado, integrando el costo por la materia prima (considerando precio del mercado), el costo de energía del molino y la mano de obra, fabricando un promedio de 8 a 10 ladrillos por un periodo de ocho horas, contando con actividades previas requeridas como el molido de la merma y las cantidades pesadas de los materiales. El costo de los ladrillos A y B oscila entre los 10.81 y 12.46 respectivamente.

El uso del ladrillo B, como se comentó anteriormente, se sugiere que sea no estructural por lo que el costo estaría por debajo del block macizo ligero.

Es importante mencionar que se debe analizar estructuralmente el comportamiento de los muros y determinar si existe la necesidad de incluir elementos de refuerzo y/o elementos de restricción de los bordes laterales y superior para evitar el volteo del muro fuera de su plano. 
La utilización de la merma de EVA en la elaboración de ladrillos permite dar una alternativa de uso de los residuos para disminuir el impacto ambiental por su disposición final. Además, al ser un material inflamable, su almacenamiento temporal genera riesgo en la seguridad de los trabajadores y la infraestructura de la empresa.

\section{Conclusiones}

Se obtuvo un material de construcción para uso no estructural, elaborado con residuos de merma de la empresa que fabrica espumas reticuladas, con el fin de ir disminuyendo la acumulación y los riesgos que se generan.

Se obtuvo dos pruebas con diferentes concentraciones de los materiales y un blanco de referencia: la prueba de ladrillo A tuvo concentraciones de tepojal, cemento, arena y merma, para la prueba de ladrillo B solamente fue de tepojal, cemento y merma, eliminando la arena.

Se evaluaron las dos pruebas y el blanco de referencia, de acuerdo a las especificaciones de la normatividad, y de acuerdo a los resultados obtenidos el ladrillo B podría desempeñar un comportamiento eficiente al ser empleado como material de construcción para el uso no estructural soportando cargas menores a una tonelada y absorbiendo menor cantidad de agua.

Con un costo unitario por ladrillo B de \$13.1 comparado con un ladrillo convencional en el mercado de $\$ 15.38$ está por debajo del precio.

\section{Agradecimiento}

Agradecemos al Lic. Benito Bastida García por su valiosa participación en este trabajo.

\section{Referencias}

Bouazza y Zornberg. (2019). Geosintéticos en Rellenos sanitarios. Sociedad Internacional de Geosinteticos (IGS).

Hax; et al. (2000). Utilización de residuos de EVA provenientes de industrias calzadistas en el aislamiento de ruidos de impacto de edificaciones. En P. Hax, \& G. A. Pizzutti. Brasil: UFSM.
Melo y Mendoca. (2017). Cement blocks wit EVA waste for extensive modular green roof: contribution of components in thermal insulation . Revista IBRACON, Vol. 10.

Melo y Silva. (2013). Bloques de hormigón ligero con áridos reciclados de EVA:. En E. P. A. B. De Melo, Materiales de Construcción (págs. 312, 479-495). Brasil: Universidad Federal de Paraíba.

NMX-C-036-ONNCCE. (2013). Industria de la Construcción - Mampostería - Resistencia a la Compresión de Bloques, Tabiques O ladrillos y Tabicones y Adoquines - Método de Ensayo.

NMX-C-037-ONNCCE. (2013). Industria de la Construcción - Mampostería - Determinación de la Absorción Total y la Absorción Inicial de Agua en Bloques, Tabiques $\mathrm{O}$ ladrillos y Tabicones - Método de Ensayo.

NMX-C-038-ONNCCE. (2013). ndustria de la Construcción - Mampostería - Determinación de las Dimensiones de Bloques, Tabiques $\mathrm{O}$ ladrillos y Tabicones - Método de Ensayo.

NMX-C-441-ONNCCE. (2005). Industria de la Construcción - Mampostería - Bloques, Tabiques o Ladrillos y Tabicones Para Uso No Estructural.

Zattera, et, al. (2005). Caracterización de residuos de copolimeros de etileno. vinil. acetato (EVA). En B. O. Zattera A. Brasil: Ciencia y Tecnología. 